\title{
Comparison of the Shikani Optical Stylet and Macintosh Laryngoscope for Orotracheal Intubation with Topical Anesthesia in Critically III Patients
}

\author{
Zhipeng Li, Meirong Wang, Qiong Wang, Chuiliang Liu* \\ Department of Anesthesiology, Chancheng Center Hospital, Foshan, PR China \\ Email address: \\ 476140516@qq.com (Chuiliang Liu) \\ ${ }^{*}$ Corresponding author \\ To cite this article: \\ Zhipeng Li, Meirong Wang, Qiong Wang, Chuiliang Liu. Comparison of the Shikani Optical Stylet and Macintosh Laryngoscope for \\ Orotracheal Intubation with Topical Anesthesia in Critically Ill Patients. Clinical Medicine Research. Vol. 7, No. 4, 2018 , pp. 84-91. \\ doi: 10.11648/j.cmr.20180704.11
}

Received: June 17, 2018; Accepted: August 29, 2018; Published: September 28, 2018

\begin{abstract}
Background: Emergency airway management outside operating room (OR) is associated with a higher incidence of difficult intubations. The Shikani optical stylet (SOS) has been shown to improve first-attempt success rates compared with the classic Macintosh laryngoscope (ML) in patients with difficult airway inside the operating room (OR). Objective: In this randomized controlled clinical trial, the effectiveness of using a SOS as the primary intubation device was assessed during urgent endotracheal intubations (UEI) in conscious, critically ill patients outside the OR. Method: Sixty conscious critically ill patients from May 2018 to March 2018 were randomly assigned to undergoing intubation guided by the SOS (group S, n = 30) or the Macintosh laryngoscope (group $\mathrm{M}, \mathrm{n}=30$ ) in ChanCheng Center Hospital,. After application of airway topical anesthesia and intravenous sedatives, endotracheal intubations were performed. The primary measured outcome was first-attempt intubation success rate. Secondary outcomes included ultimate success, the number of attempts, the duration of intubation, and the adverse effects or complications of intubation. Result: The rate of successful intubation at the first-attempt was superior with the SOS as compared with the $\operatorname{ML}(93.3 \%$ vs $63.3 \%, P=0.005)$, the average number of attempts required for successful tracheal intubation $(1.0 \pm 0.3$ vs $1.5 \pm 0.8, \mathrm{P}=0.004)$, the duration of intubation $(18.8 \pm 11.9$ vs $26.8 \pm 15.6, \mathrm{P}=0.028)$, and the incidences of hemorrhage in the oropharyngeal mucosa $(0 \mathrm{vs} 28.6 \%, \mathrm{P}=0.006)$ were all improved significantly with use of the SOS compared with the ML. Conclusion: This study suggested SOS could be used as the effective device when UEIs are performed by experienced operators in conscious, critically ill patients outside the operating room.
\end{abstract}

Keywords: Airway Management, Intubation, Intratracheal, Critical Illness, Laryngoscope, Optical Stylet

\section{Introduction}

Hypoxemia due to airway obstruction or respiratory inhibition often threatens the lives of critically ill patients. Endotracheal intubation is a lifesaving procedure frequently performed in the emergent treatment of critically ill patients with respiratory dysfunction [1, 2]. Traditionally, intratracheal intubation has been performed with a Macintosh laryngoscope (ML), which requires alignment of the oral, pharyngeal and laryngeal axes to allow direct visualization of the glottic inlet. Emergency airway management in critically ill patients outside operating room $(\mathrm{OR})$ is associated with a higher incidence of difficult intubations, ranging from $10 \%$ to $22 \%$, and increases the risk of respiratory and hemodynamic complications, including death [3-8].

The Shikani optical stylet (SOS) is a relatively new airway management tool originally designed as an aid for difficult intubation and composed of an optical stylet with a malleable shaft and a lightweight battery powered LED light source (Figure 1) [9, 10]. The SOS has been shown to increase first-attempt success rate for tracheal intubation in patients with a difficult airway in elective OR [11-15]. The usefulness of the SOS for urgent endotracheal intubations (UEIs) in 
conscious, critically ill patients outside the OR has not been well studied.

This study assessed the effectiveness of using a SOS as the primary intubating device in conscious, critically ill patients. It was hypothesized that intubation using a SOS was superior to that of a ML with regard to first-attempt success rate, average number of attempts, duration of the intubation and intubation-related complications when UEI was performed by experienced anesthesiologists.

\section{Patient and Methods}

\subsection{Ethical Approval}

The Clinical trial registration number is ChiCTR1800015355. The study was carried out from May 2018 to March 2018 at Chancheng Center Hospital in Foshan in China. The study protocol was approved by the Clinical Ethics Committee at Chancheng Center Hospital, Foshan, China (Ethical Committee approval date: 1/03/2018, ref no. 2018 Comparison of the Shikani optical stylet and Macintosh laryngoscope for orotracheal intubation in awake patients) and written informed consent.

\subsection{Patients}

A total of 60 conscious, critically ill adult patients with retained swallowing and gag reflexes underwent emergence intubation in order to assistant ventilation by respiratory machine [16] were enrolled into the study. All patients presented with indications for tracheal intubation and were intubated at bedside. Written informed consent was signed by the patient's surrogate decision makers prior to initiating procedure. Patients were excluded who were younger than 16 year, refused to tracheal intubation, unconscious or the mouth opening less than $1.5 \mathrm{~cm}$. Patients with tumors polyps, abscesses or bleeding in the airway were also excluded. Patients were excluded if they refused to tracheal intubation.

\subsection{Intubation Procedure}

Based on a computer-generated random sequence from IBM SPSS Statistics for Windows, version 22.0 (IBM Corporation, released 2014, Armonk, New York, USA) by an assistant who was not involved in the study, patients were randomly assigned to two groups of 30 . Patients in the control group had their tracheas intubated with the ML (the ML group), whereas in the second group intubation was with the SOS (the SOS group).

Mean arterial pressure (MAP), heart rate (HR) and lead II electrocardiograms (ECG), peripheral $\mathrm{O}_{2}$ saturations $\left(\mathrm{SpO}_{2}\right)$ were measured with a multifunction monitor (Spacelabs, Spacelabs Medical, Issaquah, Washington, USA). Patients were placed in the supine position with neutral head and flexed neck over a 7-cm thick pillow and pre-oxygenation with $50 \%$ oxygen through an oxygen face mask. Any secretions, blood, or vomits in the mouth were promptly cleared prior to the intubation. Airway topical anesthesia was performed via the "spray as you go" strategy [17]. Specifically, 2 - $3 \mathrm{~mL} \mathrm{2 \%} \mathrm{lidocaine} \mathrm{was} \mathrm{sprayed} \mathrm{from} \mathrm{the}$ mouth into the pharynx through a $15-\mathrm{cm}$ specially designed fenestrated epidural catheter. For patients who were restless or intolerant to intubation, low dose sedative and analgesic drugs, such as $2 \mathrm{mg}$ morphine or $1 \mathrm{mg}$ midazolam, were administered and repeated if necessary. All patients kept spontaneous respiration. Tracheal intubation was performed when patients showed no facial expression or strong reactions to placement of SOS or ML. Endotracheal tubes were used with inner diameter (ID) $7.0 \mathrm{~mm}$ for female and ID $7.5 \mathrm{~mm}$ for male patients. In the SOS group, an endotracheal tube was loaded with the J-type SOS (Clarus Medical, Minneapolis, MN, USA) and tracheal intubation was implemented using the "lighted stylet intubation method" [18] or "neck light transmittance" [19] combined with ocular scope inspection. Patients were asked to open their mouth, or patients' mouths were opened by the left hand of the operator. The SOS was held in the right hand with light switching on, the operator inserted and advanced the SOS along the natural curvature of the mouth to keep it in the oropharyngeal midline. Meanwhile, the brightest spot was identified in the anterior neck wall. When the brightest spot was located at the cricothyroid membrane or projected caudally toward the suprasternal notch, it indicated that the tip of the SOS were pointed toward the glottis or the tip of that had passed the glottis, while the glottis or tracheal ring could be observed through the ocular scope (Figure 1). The tracheal tube was immediately pushed gently into the trachea with the left hand and the SOS was withdrawn. Then the tracheal tube was connected to the breathing circuit. In the ML group, the tip of the endotracheal tube was bended into a J-shape with a copper wire to facilitate its entry into the glottis. Patients opened their mouths with their head tilted back and downward. Their lower jaws were raised so that three axes were kept in a line, the glottis was directly exposed using the ML (Schucman 5439D; Truphatek International, Ltd., Israel). All patients were auscultated to determine the depth of the tube after intubation and they were sedated and mechanically ventilated. 

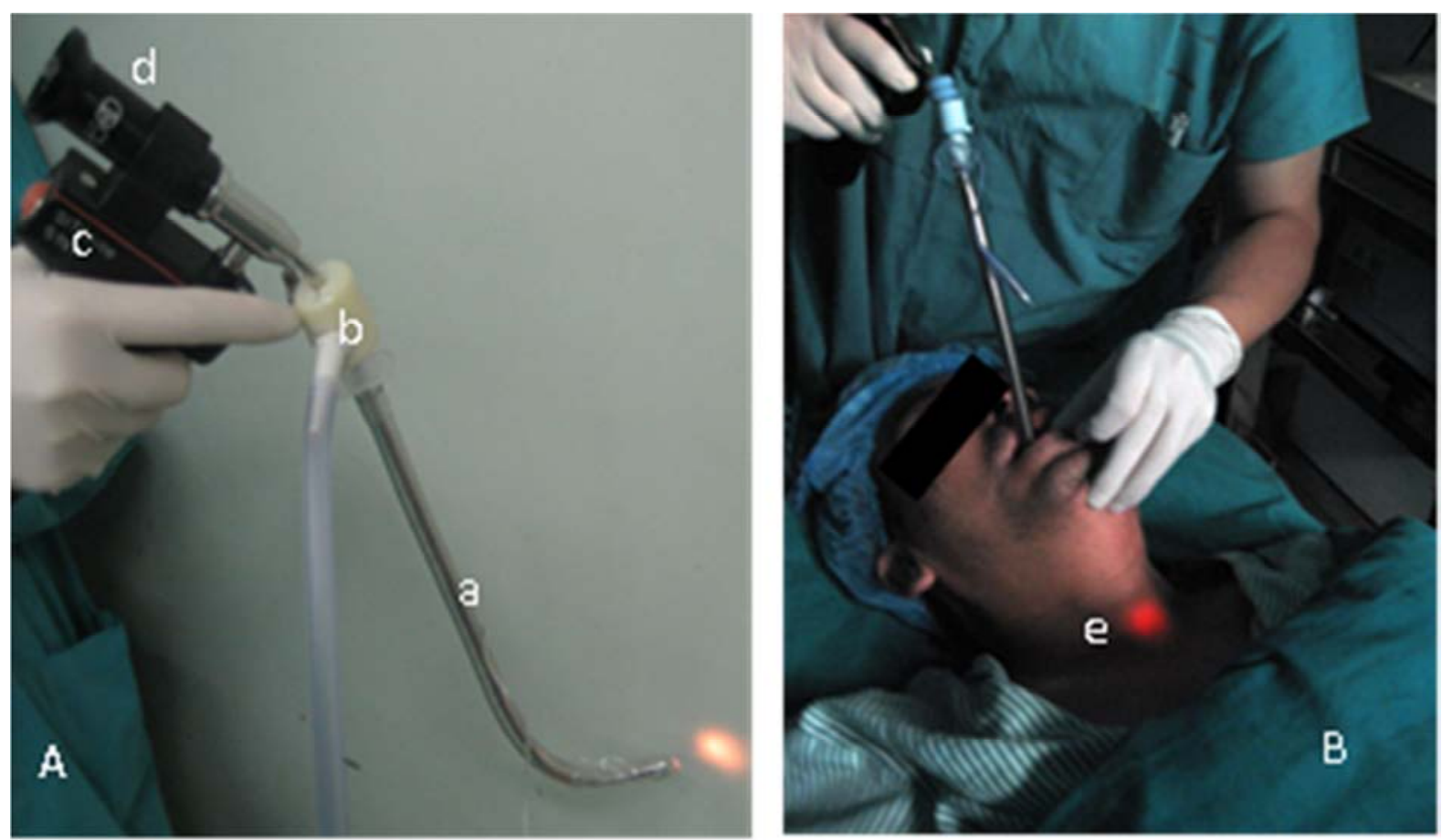

Figure 1. Endotracheal intubation with the Shikani optical stylet (SOS) A: The SOS is preloaded with an endotracheal tube. a: J-type malleable shaft; b: endotracheal tube fixation device with an oxygen delivery port on the side; $c$ : handle, built-in battery; d: ocular scope. B: The SOS intubation technique. The light spot of SOS is below the thyroid cartilage in the middle of the anterior neck wall. The SOS tip can be confirmed by the ocular scope. e: the light spot.

\subsection{Clinical Outcome Assessment}

Two anesthetists who had more than five years experience with both devices performed all intubations. An experienced consultant anesthetist, who was certified in advanced airway life support, performed the airway management for all the study subjects. All data were documented by a study nurse and analysed by a person who was blinded to the study. Standard pre-operative difficult airway predictors, such as the Mallampati score, the thyromental distance, limited neck extension, mouth opening and the body mass index $(\mathrm{kg} / \mathrm{m} 2)$ in all patients were evaluated before intubation by the consultant anesthetist. ECG, MAP, $\mathrm{HR}, \mathrm{SpO}_{2}$ were recorded before and $1 \mathrm{~min}$ after successful endotracheal intubation. The intubation attempts, the duration of intubation, the number of patients with failed intubations, the causes of failure were also recorded respectively. Failed intubation was defined as unsuccessful intubation after three attempts or an operation time that lasted more than $10 \mathrm{~min}$. Another different intubation device was used if patients failed to be intubated by the equipment arrangement in advance. The duration of intubation was defined as the time from placement of the ML or the SOS into the mouth to successful insertion of the endotracheal tube into the trachea. The number of patients whose intubations took $<15 \mathrm{sec}, 15-30 \mathrm{sec}$ and $>30 \mathrm{sec}$ were calculated.

The primary measured outcome was first-attempt intubation success rate. Secondary outcomes included average number of attempts required for successful tracheal intubation, duration of the intubation sequence, and incidence of complications.

Complications of intubation including severe cardiovascular reactions, cough, vomiting, hemorrhage of oropharyngeal cavity, sore throat and teeth damage were recorded 1 hours after extubation. Severe cardiovascular reactions were defined as fluctuations of more than $20 \%$ in MAP or heart rate or severe arrhythmia at post-intubation as compared to that at pre-intubation [20]. Mucosal hemorrhage in the oropharyngeal cavity was observed directly with the laryngoscope or fresh blood was retrieved from the oropharyngeal cavity after intubation.

\subsection{Statistical Analysis}

IBM SPSS Statistics for Windows, version 22.0 (IBM Corporation, released 2014, Armonk, New York, USA) was used to analyze the data. Test of normality was used at first, normally quantitative data were presented as means \pm standard deviation (S.D.) and using independent samples t-test, the other quantitative data were presented as means (range) and using Mann-Whitney U Test. Categorical variables were expressed as frequencies and percentages Comparisons of variables between groups were analyzed by Chi-Square test (Fisher's exact test if the number of observations obtained for analysis was small.) or rank-sum test (if more than two variables). A $P$-value of 0.05 was considered to be statistically significant. All tests are two-sided.

\section{Results}

\subsection{Patient Clinical Data}

A total of 65 eligible patients were interviewed before operation. 60 eligible patients were enrolled for randomisation. 
60 patients were analysed for haemodynamic values and TI-associated adverse events (Figure 2). There was no significant difference between the SOS and ML groups in age, gender distribution, weight, height, airway assessment and reasons for intubation (Table 1).

Assessment of intubating result. The overall success rate of TI was $100 \%$ in the SOS group and $93 \%$ in the ML group (Table 2). The rate of first-attempt success was superior with a SOS as compared with a ML (93 vs. 63\%, respectively, $P=$ 0.005) (Table 2). Patients in the SOS group required fewer attempts for successful tracheal intubation [1.0 (0.3) vs 1.5 (0.8), $P=0.004]$. The duration of intubation in the SOS group was significantly shorter than that in the ML group [18.8 (11.9) vs 26.8 (15.6), $P=0.028$ ] (Table 2). Furthermore, within $15 \mathrm{~s}$ was higher with a SOS $(67 \%$ vs $29 \%, P=0.004)$. Two patients failed to be intubated in the ML group due to difficult to expose the glottis. One patient of them was intubated through the nose with the fiberbronchoscope, the other was successfully intubated with the SOS.

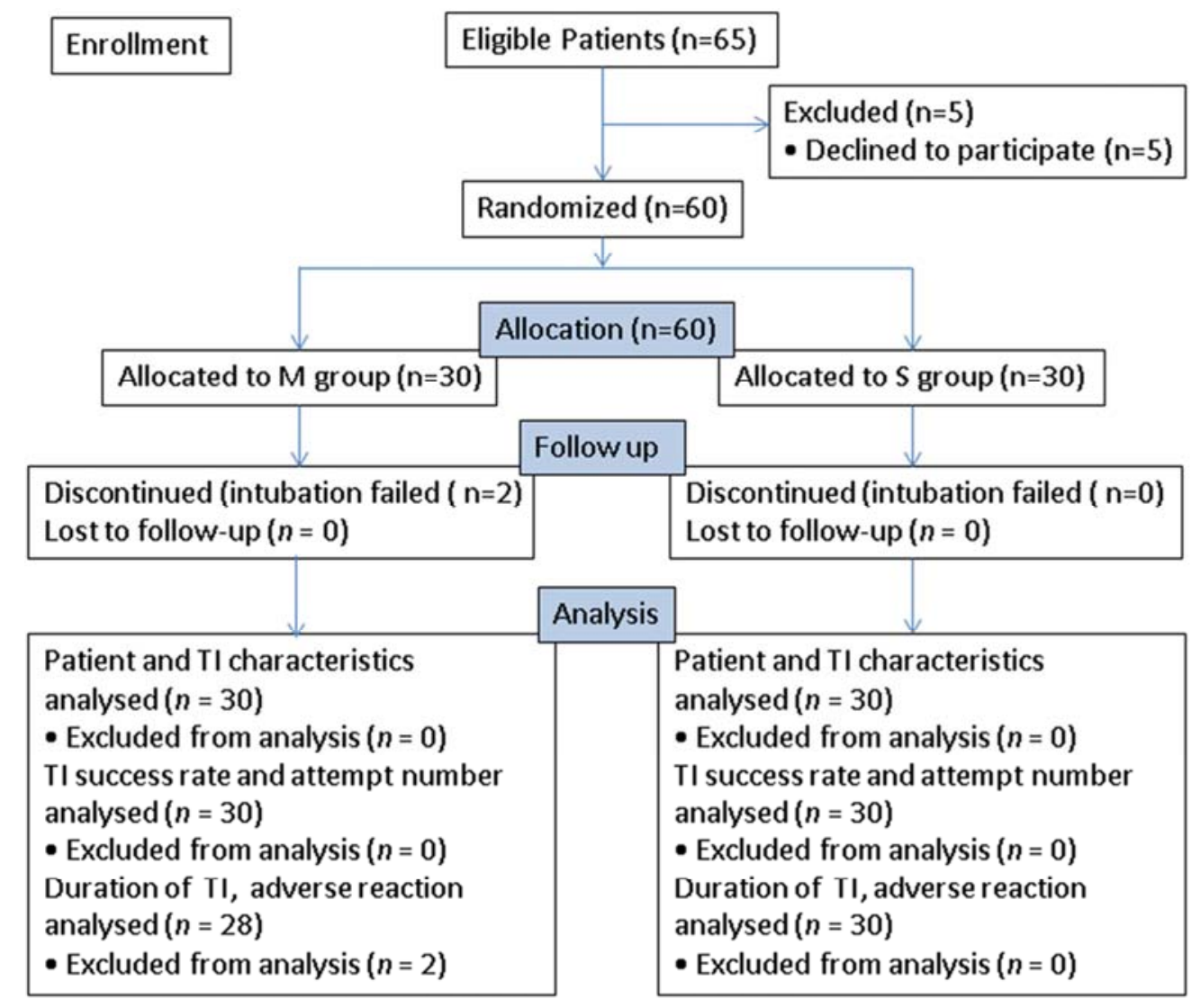

Figure 2. The study flowchart. M, Macintosh laryngoscope; S, Shikani optical stylet; TI, tracheal intubation.

Table 1. Patients demographics and intubation characteristics.

\begin{tabular}{|c|c|c|c|}
\hline Variables & ML group $(n=30)$ & SOS group $(n=30)$ & P value \\
\hline Sex, Male/Female & $19 / 11$ & $15 / 15$ & $0.4363^{*}$ \\
\hline Age, year & $56.7(14.1)$ & $57.8(13.6)$ & $0.997^{* *}$ \\
\hline Weight, kg & $60.2(9.5)$ & $58.9(10.8)$ & $0.104^{* *}$ \\
\hline Height, cm & $162.6(10.3)$ & $159.7(10.9)$ & $0.2461^{* *}$ \\
\hline BIM, $\mathrm{kgm}^{-2}$ & $23.7(4.9)$ & $24.5(4.5)$ & $0.481^{* *}$ \\
\hline Mallampati score, $1 / 2 / 3 / 4$ & $5 / 17 / 6 / 2$ & $6 / 15 / 6 / 3$ & $0.942^{* * *}$ \\
\hline Thyromental distance $<6 \mathrm{~cm}$ & $3(10.0 \%)$ & $2(6.7 \%)$ & $1.000^{\#}$ \\
\hline limited neck extension & $0(0 \%)$ & $1(3.33 \%)$ & 0.313 \\
\hline $\begin{array}{l}\text { mouth opening }<4 \mathrm{~cm} \\
\text { Reason for Intubation }\end{array}$ & $1(3.33 \%)$ & $2(6.66 \%)$ & 0.554 \\
\hline Severe trauma or surgical complications & $9(30.0 \%)$ & $13(43.3 \%)$ & $0.284 *$ \\
\hline Chronic obstructive pulmonary disease & $10(33.3 \%)$ & $6(20.0 \%)$ & $0.243 *$ \\
\hline Advanced cancer & $6(20.0 \%)$ & $7(23.3 \%)$ & $0.754 *$ \\
\hline Heart disease and other disease & $5(16.7 \%)$ & $4(13.3 \%)$ & $1.000^{\#}$ \\
\hline
\end{tabular}

Data are presented as means (SD) or actual values (percentage of group). There were no significant differences between the two groups. ML, Macintosh laryngoscope; SOS, Shikani optical stylet; BMI, body mass index. *Using Pearson Chi-Square Test; ${ }^{*}$ Using Continuous Correction Chi-Square Test; ** Using Independent Samples Test; ***Using Rank-sum Test. 
Table 2. Frequency of successful intubation and duration of intubation.

\begin{tabular}{llll}
\hline Variable & ML group $(\mathbf{n}=\mathbf{3 0})$ & SOS group $(\mathbf{n}=\mathbf{3 0})$ & P value \\
\hline Frequency of successful intubation & & & \\
Successful intubations & $28(93.3 \%)$ & $30(100 \%)$ & $0.472^{*}$ \\
First attempt & $19(63.3 \%)$ & $28(93.3 \%)$ & $0.005^{*}$ \\
Second attempt & $7(23.3 \%)$ & $2(6.7 \%)$ & $0.148^{\#}$ \\
Third attempt & $2(6.7 \%)$ & 0 & $0.472^{\#}$ \\
Failed intubations & $2(6.7 \%)$ & 0 & $0.472^{\#}$ \\
Averaging times of intubation attempt & $1.5(0.8)$ & $1.0(0.3)$ & $0.004^{* *}$ \\
Duration of intubation & $26.8(15.6)$ & $18.8(11.9)$ & $0.028^{* *}$ \\
Time for intubation, s & $8(28.6 \%)$ & $20(66.7 \%)$ & $0.004^{*}$ \\
$<15 \mathrm{~s}$ & $10(35.7 \%)$ & $8(26.7 \%)$ & $0.457^{*}$ \\
$15-30 \mathrm{~s}$ & $10(35.7 \%)$ & $2(6.7 \%)$ & $0.006^{*}$ \\
\hline $30 \mathrm{~s}$ & &
\end{tabular}

Data are presented as means (SD) or actual values (percentage of group). ML, Macintosh laryngoscope; SOS, Shikani optical stylet. "Using Continuous Correction Chi-Square Test; *Using Pearson Chi-Square Test; **Using Independent Samples Test.

The MAP in the SOS and ML group was significantly higher after intubation [82.7 (13.5) vs 99.7 (16.91) $\mathrm{P}=0.001 ; 84.9$ (9.5) 94.5 (8.91) $\mathrm{P}=0.002$ ], $\mathrm{The} \mathrm{SpO}_{2}$ in the $\mathrm{SOS}$ and ML group was increased significantly after intubation [*76.8 (10.9) 96.8 $(3.21)$ $\mathrm{P}=0.000 ; 75.4$ (12.3) 97.1 $\pm(2.91) \mathrm{P}=0.000$ ], there were no significantly differences on MAP, HR and $\mathrm{SpO} 2$ between in the SOS and ML group (Table3 ).

Table 3. $\mathrm{MAP}, \mathrm{HR}, \mathrm{SpO}_{2}$ 1min before and 1min after successful endotracheal intubation.

\begin{tabular}{lllll}
\hline & Group & 1min before intubation & 1min after intubation & P value \\
\hline \multirow{2}{*}{ MAP (mmHg) } & ML group & $82.7(13.5)$ & $99.7(16.91)^{*}$ & $\mathrm{P}=0.0001$ \\
& SOS group & $84.9(9.5)$ & $94.5(8.91)^{*}$ & $\mathrm{P}=0.0002$ \\
$\mathrm{HR}$ & ML group & $85.9 \pm(23.5)$ & $92.4(15.8)^{*}$ & $\mathrm{P}=0.2087$ \\
& SOS group & $86.2(22.8)$ & $89.4 \pm(13.4)^{*}$ & $\mathrm{P}=0.5101$ \\
$\mathrm{SpO} 2$ & ML group & $76.8(10.9)$ & $96.8 \pm(3.21)^{*}$ & $\mathrm{P}=0.0000$ \\
& SOS group & $75.4(12.3)$ & $97.1 \pm(2.91)^{*}$ & $\mathrm{P}=0.0000$ \\
\hline
\end{tabular}

Data presented as means (SD). ML, Macintosh laryngoscope; *Using Independent Samples Test.

\subsection{Adverse Events in Patients During Procedure}

The incidence of mucosal injury and hemorrhage in the oropharyngeal cavity was less with a SOS as compared with a ML (0 vs $29 \% ; P=0.006$ ). Other adverse events like severe cardiovascular reactions, acute cough, vomiting and teeth injury occurred similarly in both groups (Table 4).

Table 4. Adverse reactions during intubation

\begin{tabular}{|c|c|c|c|}
\hline Variable & ML $\operatorname{group}(n=30)$ & SOS group $(n=30)$ & $P$ value \\
\hline Severe cardiovascular reactions & $12(40 \%)$ & $11(36.7 \%)$ & $0.791 *$ \\
\hline Cough & $19(63.3 \%)$ & $18(60 \%)$ & $0.791 *$ \\
\hline Haemorrhage of oropharyngeal mucosa & $8(26.7 \%)$ & 0 & $0.008^{\#}$ \\
\hline Tooth damage & $1(3.3 \%)$ & 0 & $0.313^{* *}$ \\
\hline Sore throat & $6(20 \%)$ & $3(10 \%)$ & $0.278^{\#}$ \\
\hline
\end{tabular}

Data presented as actual values (and percentage of group). ML, Macintosh laryngoscope; SOS, Shikani optical stylet. *Using Pearson Chi-Square Test; ${ }^{*}$ Using Continuous Correction Chi-Square Test; **Using Fisher's Exact Test.

\section{Discussion}

The present study demonstrated higher rate of first-attempt success of tracheal intubation, less time required for intubation and hemorrhage complications during UEI when a SOS was used as the primary intubating device compared with a ML in awake, critically ill patients.

Airway management in the awake, critically ill patients can be very risky due to difficult anatomic features of the patient and decompensated cardiopulmonary physiology. Prior research has shown that SOS is useful in anticipated difficult airway management under general anesthesia $[13,14,20,21$, 22], while the role of SOS on managing airway in awake, critically ill patients is yet to be defined. SOS combines the benefits of the lightwand and fiberoptic bronchoscope [9, 10, 21]. The lighted spot on the anterior surface of the neck allows guidance to place the tip of the SOS into the glottis or trachea. These features can effectively shorten the time required to identify the structure of the pharynx under the ocular scope, thereby reducing the time to intubate patients. Among the few patients in whom it is difficult to identify the light spots in the neck, the position of the intubation tube can be viewed by the ocular scope to avoid a "blind" operation and to increase the 
success rate of intubation $[23,24]$. Phua et al reported the first - attempt success rate with a SOS was similar as compared with a GlideScope videolaryngoscope in anesthetic patients with a simulated difficult airway (93\% vs 97\%) [21].

In this study, awake, critically ill patients were intubated with spontaneous respiration after application of topical anesthetic and light sedation. The results identified that the first - attempt success rate with a SOS was 93\%, significantly higher as compared with a ML (63\%), which was consistent with the study of Phua. Abdellatif et al reported the first attempt success rate was $75 \%$ with flexible fiberoptic bronchoscope (FOB) and 80.6\% with Glide Scope video laryngoscopy (GVL) for awake intubation of obese patient with predicted difficult intubation [25]. Mosier et al reported the first attempt success rate was $79 \%$ with video laryngoscopy (VL) for patients in the Intensive Care Unit (ICU) [26]. One of the reasons of the higher success rate (93\%) with a SOS in our present study was that only $37 \%$ patients with predicted difficult intubation in the ML and SOS groups compared to $100 \%$ patients with predicted difficult intubation in study of Abdellatif et al [25]; Moreover, the intubations were performed by a senior anesthesia residents in our study while which were performed by a fellow or a resident of ICU in study of Mosier et al [27], which might be helpful to increase the first attempt success rate. The first attempt success rate with a ML (63\%) was similar with the study of Mosier et al [26], which was $61 \%$ with direct laryngoscopy for patients in ICU, but lower than the anesthetic patients without difficult airway in the operating room [27]. Evans et al reported that the time taken to intubate a manikin with a SOS was shorter than with a gum elastic bougie [20.8 (9.3) seconds vs 30.0 (19.8) seconds] [28]. While using the left molar approach, patients can be intubated more quickly (less than 20 seconds) [23]. The present results showed the duration of intubation with a SOS was shorter than with ML in critically ill patients with spontaneous respiration [18.8 (11.9) seconds vs 26.8 (15.6) seconds], and two thirds of the patients with a SOS completed intubation within 15 seconds $(67 \%$ vs $29 \%, P$ $=0.004)$. After topical anesthesia, most patients could cooperate with the insertion of SOS. The operators had to raise the mandibula with the left thumb to facilitate the SOS insertion in a few uncooperative patients. Because the soft tissue in the pharynx does not need to be raised to expose the glottis, SOS is well-tolerated for patients and produces a high first attempt success rate, particularly in patients with difficult airway or limited mouth opening [9, 10, 12, 20, 21]. Two patients intubated with a SOS took more than 30 seconds for intubation in this study because of obesity and short neck, which resulted in difficulty in identifying the light spot. The intubation was successfully performed by switching off the room light for easy recognition of the light spot along the anterior neck wall and locating the glottis under the ocular scope.

Compared with the flexible fiberoptic bronchoscope, the SOS has a narrow field of vision and a small depth of field. Therefore, an unskilled operator may take longer to find the glottis through the ocular scope. Patients intubated with ML exhibited strong resistance to the lifting force of the laryngoscope. Some patients had anatomic abnormalities or relatively high secretion volume in the mouth, resulting in poor glottis exposure, repeated attempts of tracheal intubation (37\%), or failed intubation (7\%). Our results suggested SOS provided more effective and efficient intubation in the awake, critically ill patients which might benefit for improving success rate of saving life. In addition, patients intubated with a SOS can be maintained in a natural supine position without an excessive backward positioning of the head and neck, reducing the risks associated with cervical spinal injury [29, 30]. Continuous oxygen supplementation through the oxygen delivery port at the proximal part of the SOS greatly increases the safety of endotracheal intubation.

The studies of Yao and Kimura have indicated use of the SOS reduces the hemodynamic response to intubation in normotensive and hypertensive patients [25, 31]. Our present results were inconsistent with them which showed that cardiovascular reactions could not be adequately and effectively controlled in both groups. Among critically ill patients whose consciousness and reflexes have not completely disappeared, stimulation of the base of the tongue and the throat directly by laryngoscope, along with stimulation of the trachea by inserting the endotracheal tube, may lead to serious complications, such as severe cardiovascular reactions, coughing, nausea and vomiting. Despite topical anesthetic with low dose sedation was used before intubation, stimulation of the trachea by inserting the tube was not completely inhibited. Takahas $\mathrm{M}$ et al reported direct stimulation of the trachea appears to be a major cause of the hemodynamic changes associated with tracheal intubation [32], the mechanosensory-induced cough reflex during intubation should also be considered [33], which 'might partly explain the incidences of severe cardiovascular reactions, coughing, was similar as compared with ML in present study.

Teeth or mucosal injury is common complications in difficult tracheal intubation, we encountered eight patients in whom oropharyngeal mucosal haemorrhage was noted and one patient whose teeth were damaged with a ML, while there was no case of oropharyngeal mucosal injury and teeth damage with a SOS. In the study of a simulated difficult airway, a lower incidence of airway mucosal injury was also noted in patients intubated with the SOS, compared with the GlideScope video laryngoscope [21]. These results suggested using SOS for intubation in awake, critically ill patients reducing the possibility of damage to the mucosa. We encountered six patients in whom sore throat was noted with a ML, while there were three cases of sore throat with a SOS. The results suggested that there were no significant difference of sore throat between these two method. More reasearch should be made before firm conclusions are to be drawn because the number of cases was limited.

There are several limitations to this study, which ought to be mentioned. First, the lack of the follow-up survey of the patients enrolled, further study to assess the prognosis and outcome of critically ill patients when endotracheal intubated with SOS is required. Second, we have compared first attempt 
success rate and complication between the SOS and the ML among experienced anesthesiologist, whether the success rate will be improved when the SOS is utilized by trained residents in the department of emergency needs further investigation. Moreover, we did not compare SOS to the other device similar in structure, e.g. Bonfis, C-MAC, which may be considered in future study.

\section{Conclusion}

In summary, this study showed that endotracheal intubation guided by the SOS leads to a higher first attempt success rate, less time for intubation and a lower incidence of airway mucosal injury as compared with the ML use.

The first attempt success rate with the SOS was higher compared with Macintosh laryngoscope, which means that the SOS can be used particularly in patients with difficult airway. For patients of obesity and short neck with difficult airway or limited mouth opening, the SOS was a better choice. The Macintosh laryngoscope should never be forgotten when we meet obese patients with short neck. The intubation was successfully performed by switching off the room light for easy recognition of the light spot along the anterior neck wall and locating the glottis under the ocular scope. If the room light could be switched off, intubation could be finished faster which resulted in convenience in identifying the light spot. The SOS reduced the possibility of damage to the mucosa when switching off the room light, which was a better choice when ML was not suitable for the patient.

The SOS is an effective alternative to the ML for tracheal intubation in awake, critically ill patients.

\section{Acknowledgements}

This work was supported by research grants from the Medical Science and Technology Program of Foshan (No. 20130841).

\section{Conflicts of Interest/Disclosures}

All the authors do not have any possible conflicts of interest.

\section{Authors' Contributions}

C. L. participated in conception and design of the study. Z. L. and M. W carried out the experimental work and data collection. Q. W. contributed to analysis and interpretation of data. Y. L. drafted the article. All the authors read and approved the final article.

\section{References}

[1] Higgs A, Cook T M, Mcgrath B A. Airway management in the critically ill: the same, but different. British Journal of Anaesthesia, 117 (suppl_1):i5-i9, 2016.
[2] Nolan JP, Soar J. Airway techniques and ventilation strategies. Curr Opin Crit Care 14: 279-286, 2008.

[3] Kim W Y, Kwak M K, Ko B S, et al. Factors Associated with the Occurrence of Cardiac Arrest after Emergency Tracheal Intubation in the Emergency Department. Plos One, 9 (11):e112779, 2014.

[4] Jaber S, Amraoui J, Lefrant JY, et al. Clinical practice and risk factors for immediate complications of endotracheal intubation in the intensive care unit: a prospective, multiplecenter study. Crit Care Med 34: 2355-2361, 2006.

[5] Mort TC. Emergency tracheal intubation: complications associated with repeated laryngoscopic attempts. Anesth Analg 99: 607-613, 2004.

[6] Higgs A, Mcgrath B A, Goddard C, et al. Guidelines for the management of tracheal intubation in critically ill adults. British Journal of Anaesthesia, 120 (2):323-352, 2018.

[7] Le Tacon S, Wolter P, Rusterholtz T, et al. Complications of difficult tracheal intubations in a critical care unit. Ann Fr Anesth Reanim 19: 719-724, 2000.

[8] Martin LD, Mhyre JM, Shanks AM, et al. 3, 423 emergency tracheal intubations at a university hospital: airway outcomes and complications. Anesthesiology 114: 42-48, 2011.

[9] Liem E, Bjoraker D, Gravenstein D. New options for airway management: intubating fiberoptic stylets. Br J Anaesth 91: 408-418, 2003.

[10] Shikani AH. New "seeing" stylet-scope and method for the management of the difficult airway. Otolaryngol Head Neck Surg 120: 113-116, 1999.

[11] Stricker P, Fiadjoe JE, McGinnis S. Intubation of an infant with Pierre Robin sequence under dexmedetomidine sedation using the Shikani Optical Stylet. Acta Anaesthesiol Scand 52: 866-867, 2008.

[12] Langeron O, Amour J, Vivien B, Aubrun F. Clinical review: management of difficult airways. Crit Care 10: 243, 2006.

[13] Jansen AH, Johnston G. The Shikani Optical Stylet: a useful adjunct to airway management in a neonate with popliteal pterygium syndrome. Paediatr Anaesth 18: 188-190, 2008.

[14] Shukry M, Hanson RD, Koveleskie JR, Ramadhyani U. Management of the difficult pediatric airway with Shikani Optical Stylet. Paediatr Anaesth 15: 342-345, 2005.

[15] Tan Y, Tang W, Anesthesiology D O. The application of Shikani Seeing Optical Stylet in difficult airway intubation in children. Journal of Modern Medicine \& Health, 2014.

[16] Hernandez G, Peña H, Cornejo R, et al. Impact of emergency intubation on central venous oxygen saturation in critically ill patients: a multicenter observational study [J]. Critical Care, 2009, 13:R63.

[17] Xue FS, Liu HP, He N, et al. Spray-as-you-go airway topical anesthesia in patients with a difficult airway: a randomized, double-blind comparison of $2 \%$ and $4 \%$ lidocaine. Anesth Analg 108: 536-543, 2009.

[18] Valverde C M D L L, López F G, Sánchez A T, et al. Lighted stylet tracheal intubation. Actualizaciones En Anestesiologia Y Reanimacion, 14:123-126, 2004. 
[19] Williams B A, Kentor M L, Williams J P, et al. PACU bypass after outpatient knee surgery is associated with fewer unplanned hospital admissions but more phase II nursing interventions.[J]. Anesthesiology, 2002, 97 (4):981.

[20] Yang D, Tong SY, Jin JH, et al. Shikani optical stylet-guided intubation via the intubating laryngeal airway in patients with scar contracture of the face and neck. Chin Med Sci J 28: 195-200, 2013.

[21] Phua DS, Mah CL, Wang CF. The Shikani optical stylet as an alternative to the GlideScope ${ }^{\circledR}$ videolaryngoscope in simulated difficult intubations--a randomised controlled trial. Anaesthesia 67: 402-406, 2012.

[22] Young CF, Vadivelu N. Does the use of a laryngoscope facilitate orotracheal intubation with a Shikani Optical Stylet? Br J Anaesth 99: 302-303, 2007.

[23] Yao YT, Jia NG, Li CH, et al. Comparison of endotracheal intubation with the Shikani Optical Stylet using the left molar approach and direct laryngoscopy. Chin Med J (Engl) 121: 1324-1327, 2008.

[24] Xue FS, He NL, Jian H, et al. Awake orotracheal intubation with the Bonfils fibrescope in patients with a difficult airway. Eur J Anaesthesiol 27: 305-306, 2010.

[25] Abdellatif AA, Ali MA. GlideScope videolaryngoscope versus flexible fiberoptic bronchoscope for awake intubation of morbidly obese patient with predicted difficult intubation. Middle East J Anaesthesiol 22: 385-392, 2014.

[26] Mosier JM, Whitmore SP, Bloom JW, et al. Video laryngoscopy improves intubation success and reduces esophageal intubations compared to direct laryngoscopy in the medical intensive care unit. Crit Care 17: R237, 2013.

[27] Griesdale DE, Liu D, McKinney J, Choi PT. Glidescope $\AA$ video-laryngoscopy versus direct laryngoscopy for endotracheal intubation: a systematic review andmeta-analysis. Can J Anaesth 59: 41-52, 2012.

[28] Evans A, Morris S, Petterson J, et al. A comparison of the Seeing Optical Stylet and the gum elastic bougie in simulated difficult tracheal intubation: a manikin study. Anaesthesia 61: 478-481, 2006.

[29] Turkstra TP, Pelz DM, Shaikh AA, et al. Cervical spine motion: a fluoroscopic comparison of Shikani Optical Stylet vs Macintosh laryngoscope. Can J Anaesth 54: 441-447, 2007.

[30] Dupanovic M, Fox H, Kovac A. Management of the airway in multitrauma. Curr Opin Anaesthesiol 23: 276-282, 2010.

[31] Aghdaii N, Azarfarin R, Yazdanian F, et al. Cardiovascular responses to orotracheal intubation in patients undergoing coronary artery bypass grafting surgery. Comparing fiberoptic bronchoscopy with direct laryngoscopy. Middle East Journal of Anesthesiology, 20:833-8, 2010.

[32] Jeon Y G, Park J, Kim M H, et al. Hemodynamic response to tracheal intubation and postoperative pharyngeal morbidity using GlideScope ${ }^{\circledR}$, Lightwand and Macintosh laryngoscopes during remifentanil infusion. 12:342-347, 2017.

[33] Brooks S M. Perspective on the human cough reflex. Cough, 7:10, 2011 